\title{
Intralabyrinthine Cholesterol Granuloma with Rapid Progression After Acute Hemorrhage, A Common Origin?
}

\author{
Marta Navarro Fornas ${ }^{1 *}$, Carlos de Paula Vernetta ${ }^{1,2}$, Fernando Mas Estellés ${ }^{3}$, Abel Guzmán Calvete ${ }^{1}$, Laura \\ Cavallé Garrido ${ }^{1,2}$ and Miguel Armengot Carceller ${ }^{1,2,4}$ \\ ${ }^{1}$ Department of Otorhinolaryngology, Hospital Universitario y Politécnico La Fe, Valencia, Spain \\ ${ }^{2}$ University of Valencia, Spain \\ ${ }^{3}$ Department of Radiology, Hospital Universitario y Politécnico La Fe, Valencia, Spain \\ ${ }^{4}$ Instituto de investigación sanitaria La Fe, Research institute in Valencia, Spain
}

*Corresponding author: Marta Navarro Fornas, Department of Otorhinolaryngology, Hospital Universitario y Politécnico La Fe, Valencia, Spain.
Received Date: August 30, 2021

Published Date: September 22, 2021

\section{Abstract \\ We present the case of a cholesterol granuloma after acute hemorrhage in the inner ear with rapid progression to adjacent structures such as vestibular aqueduct or inner auditory canal. After 6 months of its total resection, the patient has had no local recurrence.}

Keywords: Cholesterol granuloma, Intralaberinthine hemorrhage, Hypoacusia, Peripheral vertigo, Petrous apex

Abbreviations: Computed tomography (CT); Magnetic resonance image (MRI)

\section{Introduction}

Cholesterol granuloma is the most common benign nonneoplasic lesion of the petrous apex. The ethiology of this phenomenon is based on the entrance of blood into a mucosal space. It is believed that anaerobic degradation of blood products, including cholesterol crystals, may provoke a foreign body giant cell reaction that induce the cyst formation [1]. Once the cysticgranuloma is present, patients may suffer from cocleo-vestibular symptoms, as a consequence of the location of the lesion. However, sometimes they can be asymptomatic [2]. After suffering an acute inner ear hemorrhage, our patient showed clear signs of audiologic and vestibular affection that persisted for 8 months. The lack of improvement made necessary the realization of new complementary tests to get to our new diagnosis.

\section{Case Presentation}

A 40-year-old man presented in our hospital referring sudden right-sided hypoacusia, tinnitus and vertigo. Audiologic and vestibular tests confirmed cophosis and vestibular disfunction of the affected side. Radiological findings on MRI showed hiperintensity signal in T1 and hypointensity in T2, suggesting acute intralaberinthine hemorrhage as the main cause of the symptoms. The origin of this hemorrhage remained unknown. After 8 months of maintained cocleo-vestibular disfunction, a new MRI showed an extense occupation of the petrous portion of the right temporal bone, non-existent on the initial image. This lesion associated high intensity signal in T1 and T2. The same content could be found occupying the cochlear compartments, vestibule and superior semicircular canal as a result of extended inner ear fistulization of cholesterol granuloma. More precisely, to perilinfatic space (Figure 1).

Axial T1 and T2-weighted MRI shows a space-occupying lesion in the right petrous apex $(*)$, with its characteristic hyperintensity suggesting cholesterol granuloma. It can be appreciated the 
extension of the mass to the cochlea and superior semicircular canal (arrows), with marked signal asymmetry in $\mathrm{T} 1$ regarding the left side.

The extension to the postero-medial portion of the right temporal bone was dilucidated by CT, with anterior limit on the yugular plate. The mass eroded its medial wall, the limit with the vestibular aqueduct and posterior wall of internal auditory canal. Total surgical resection of the cholesterol granuloma was achieved with a translabyrinthine approach. Posteriorly, surgery was completed with complete obliteration of the ear cavity with fat tissue. Our patient remains clinically stable and without relapses after 6 months post-surgically (Figure 2).

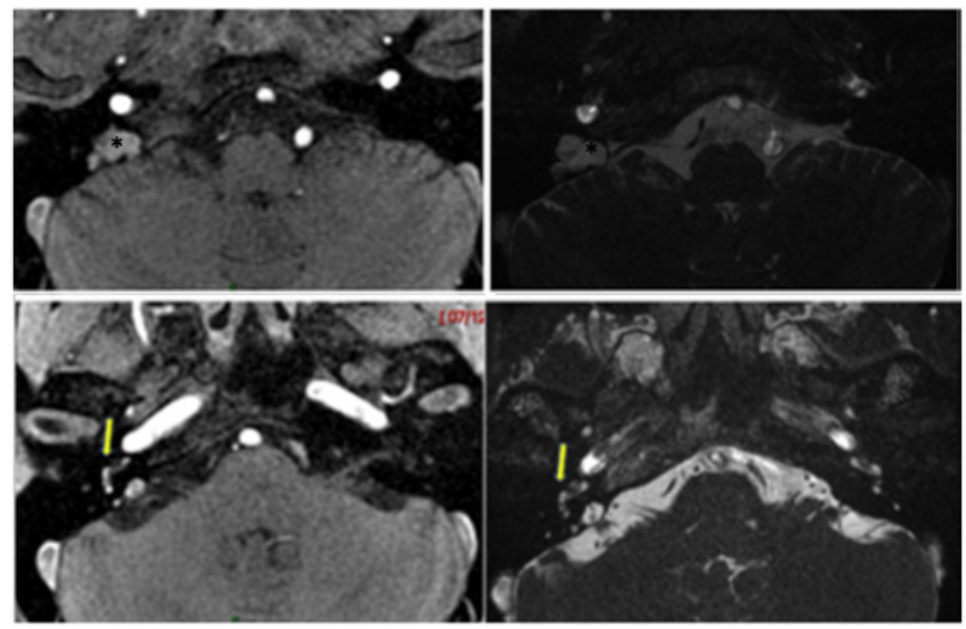

Figure 1: Preoperative imaging.

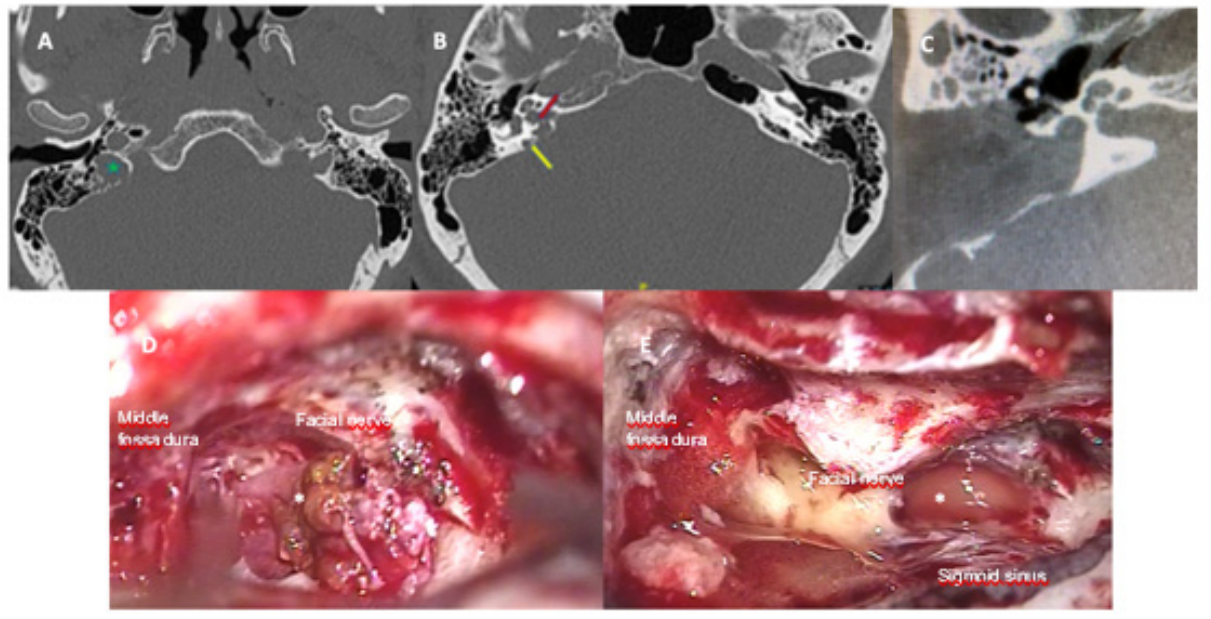

Figure 2: Preoperative and postoperative imaging. Intraoperative findings.

Axial plain computed tomography (CT) of the temporal bone before (A and $B$ ) and after surgical intervention (C). It reveals the presence of an expansive lesion in the postero-medial region of the temporal bone (star), eroding bone limits with the medial wall, the vestibular aqueduct (yellow arrow) and internal auditory canal (red arrow). A direct vision of the cholesterol granuloma (asterisk) is obtained during surgery (D), as well as the residual space after its complete resection (E).

\section{Discussion}

The incidence of petrous apex cholesterol granuloma has been estimated at 0.6 cases per 1 million population. Diagnosis is fulfilled by computed tomography (CT) or magnetic resonance (MR), being typical findings of cholesterol granuloma the hiper-intensity in T1/ T2. These characteristics will help in differential diagnosis with other pathologies of the petrous apex [1,2]. Despite its histological benign features, cholesterol granuloma can be dangerous due to its agressive tendency to grow and invade proximal spaces. For this reason, we need to consider surgery and total resection of the lesion as its main treatment. The objective is to preserve integrity of the surrounding structures, and prevent further extension to the endocraneal space. For surgery indication and election of the type of approach, we must take into account the size of the lesion, its location and the patient's age. 


\section{Acknowledgement}

None.

\section{Conflict of Interest}

The authors declare no potential conflicts of interest.

\section{Funding}

The authors received no specific funding for this work.

\section{References}

1. Isaacson B (2015) Cholesterol granuloma and other petrous apex lesions. Otolaryngol Clin North Am 48(2): 361-373.

2. Kuruma T, Tanigawa T, Uchida Y, Tetsuya O, Ueda H (2017) Large Cholesterol Granuloma of the Middle Ear Eroding into the Middle Cranial Fossa. Case Rep Otolaryngol (3): 1-5. 